\title{
A Requirements-Driven Optimization Method for Acoustic Liners using Analytic Derivatives
}

\author{
Jeffrey J. Berton* \\ NASA Glenn Research Center, Cleveland, Ohio 44135 \\ and \\ Leonard V. Lopes ${ }^{\dagger}$ \\ NASA Langley Research Center, Hampton, Virginia 23681
}

\begin{abstract}
More than ever, there is flexibility and freedom in acoustic liner design. Subject to practical considerations, liner design variables may be manipulated to achieve a target attenuation spectrum. But characteristics of the ideal attenuation spectrum can be difficult to know. Many multidisciplinary system effects govern how engine noise sources contribute to community noise. Given a hardwall fan noise source to be suppressed, and using an analytical certification noise model to compute a community noise measure of merit, the optimal attenuation spectrum can be derived using multidisciplinary systems analysis methods. In a previous paper on this subject, a method deriving the ideal target attenuation spectrum that minimizes noise perceived by observers on the ground was described. A simple code-wrapping approach was used to evaluate a community noise objective function for an external optimizer. Gradients were evaluated using a finite difference formula. The subject of this paper is an application of analytic derivatives that supply precise gradients to an optimization process. Analytic derivatives improve the efficiency and accuracy of gradient-based optimization methods and allow consideration of more design variables. In addition, the benefit of variable impedance liners is explored using a multi-objective optimization.
\end{abstract}

\section{Nomenclature}

$A$

$C$

$D$

$f$

$G$

$l$

$L$

$O$
$=$ enclosed area of suppression spectrum

$=$ tone correction factor

$=$ duration correction factor

$=$ frequency

$=$ ground reflection factor

$=$ narrowband noise level

$=$ noise level

$=$ optimization objective function $r$

$R$

$w$

$x$

$\alpha$ Subscripts:

em $=$ emitted

rec $=$ received

\section{Introduction}

$\mathrm{T}$

HE most common type of acoustic treatment found in modern turbofan engine inlets and bypass exhaust ducts is the resistive facesheet resonator. Resonators generate destructive interference that reduces the level of noise propagating through a duct. A conventional resonator consists of a perforated sheet laid over cavities constructed from a honeycomb core. Subject to practical structural, maintenance and environmental considerations, liner design variables can be manipulated to achieve a target attenuation spectrum. Principal liner design variables are facesheet porosity (determining the absorption peak level) and the honeycomb cavity depth (determining the absorption peak frequency). One, two, or even three layers of these structures are found in practice. Layers are combined to create a

\footnotetext{
*Aerospace Engineer, Propulsion Systems Analysis Branch, MS 5-11, senior member AIAA.

${ }^{\dagger}$ Research Aerospace Engineer, Aeroacoustics Branch, MS 461, member AIAA.
} 
robust attenuation spectrum that covers a wide range of frequencies. A schematic of a single degree of freedom perforate-over-honeycomb liner is shown in Figure 1.

Historically, liner design variables were selected to maximize the attenuation of modes exiting a duct (e.g., [2, $3]$ ), optimized to ensure that certain spinning modes were cut off [4], or targeted simply at a particularly problematic fan interaction tone [5]. But looking to the future, industrial innovations such as additive manufacturing are enabling new liners with multiple degrees of freedom. Cavities of intricate geometry can be built up from a rigid backplate using additive techniques. Complex cavities consisting of tunnels with straight segments and sharp turns can be constructed that are not feasible using conventional manufacturing processes. New adaptive and active liners are being investigated as well. Since the acoustic signature of a fan changes with engine power setting and flight condition, the noise source that liner designers aim to suppress is a moving target. A dynamically-changing source may be more effectively suppressed with active and adaptive liners having variable impedance. Gaeta and Ahuja [6], for example, experimented with tunable acoustic liners in 1998. Now, shape-memory materials and other techniques are enabling morphing, more-tunable liner geometries that can adjust to a changing noise source by

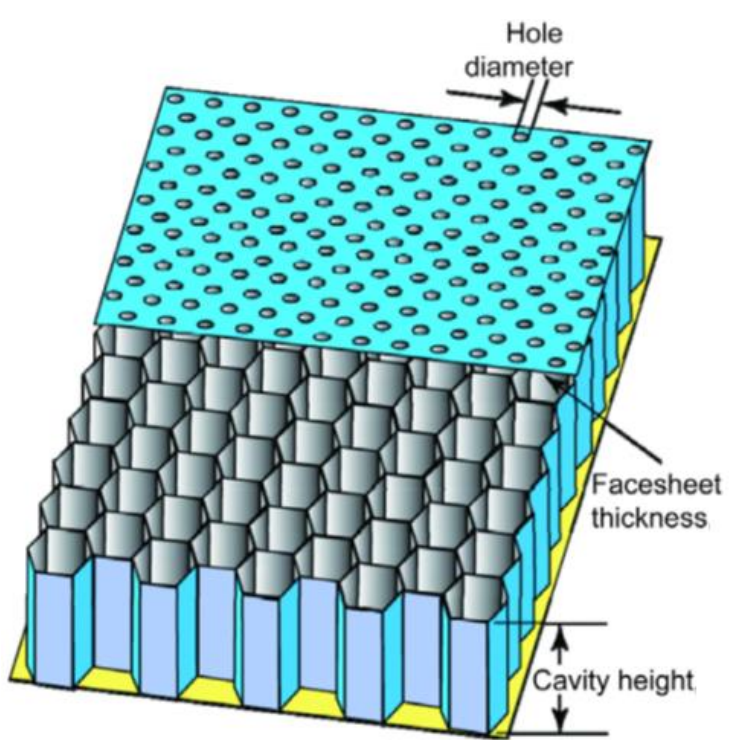

Figure 1. Single degree of freedom, perforate-overhoneycomb liner (Graphic reproduced from [1]). tailoring its suppression spectrum.

To the point, today there is greater flexibility and freedom in liner design. More than ever, liner design variables can be manipulated to achieve a very specific target attenuation spectrum. But characteristics of the ideal attenuation spectrum can be difficult to know. Hardwall fan noise character varies dramatically with engine operation, size, architecture and design intent. The "best" liner attenuation spectrum varies by application and with the metrics used to measure it.

The ideal liner perhaps may be one that can be tuned to minimize community noise measured at the three certification locations (regulated by ICAO's Annex 16 [7], or its FAA equivalent, Part 36 [8]). Propagation of an engine noise source to an observer on the ground is a complex physical phenomenon, and the process of measuring it with relevant metrics while abiding by airworthiness regulations adds more complexity. Many multidisciplinary system effects govern how engine noise sources contribute to community noise. Thus, accurately predicting community noise using analytical methods is a daunting prospect. Despite this, aircraft system noise prediction is an analytically tractable problem. Indeed, liners have been designed using local noy-weighting [9] or certification noise metrics as a minimization objective (e.g., [10-12]). But these methods do not appear to have been developed using configurable, open-source, freely-available, optimization software to attenuate arbitrary hardwall noise sources, nor have they been implemented using analytic derivatives.

If the shape of a liner suppression spectrum can be mathematically parameterized, then a set of independent "shape factor" design variables can be used to express suppression levels as a function of frequency. Given a hardwall noise source to suppress, it should then be straightforward to manipulate the shape factor variables using an optimizer until community noise is minimized. Once the ideal suppression spectrum is known, the geometric design and the impedance characteristics of a real acoustic liner could be derived to match it. In other words, the optimum acoustic liner design can be approached using a requirements-driven process to assist the traditional design.

A previous paper by the author [13] described this method. In it, the ideal target attenuation spectrum that minimizes noise perceived by observers on the ground was derived by manipulating spectral suppression shape factors. A simple code-wrapping approach was used to evaluate a community noise objective function for an external optimizer. When gradient-based optimizers were used, sensitivities were evaluated using a finite difference formula. A configurable method using open-source, object-oriented frameworking and optimization software was developed for this purpose as a general tool. The subject of this paper is an application of analytic derivatives to supply precise gradients to the optimization process. Analytic derivatives improve the efficiency and accuracy of the method and allow consideration of many more design variables. In addition, the benefit of variable impedance liners is explored using a multi-objective optimization.

2 of 13

American Institute of Aeronautics and Astronautics 


\section{Method of Analysis}

\section{A. System Noise Modeling}

Analytically modeling airport community noise is a complex proposition. In most systems-level prediction methods, aircraft noise sources are modeled using free-field, lossless sound pressure levels defined on an arc of constant radius. In the case of fan noise, the source is usually treated as compact, at least insofar as a distant observer is concerned. The noise levels are cast as a function of frequency, emission angle(s), flight condition, and engine state. This modeling process is shown schematically in Figure 2. The noise source subject to liner suppression can be combined with other sources nearby on the airplane or modified by other local effects before propagation to the ground. As the source is analytically flown through the air, its acoustic signature changes. From the viewpoint of a stationary observer, distance and emission angles vary as the source first approaches and then recedes. Doppler shift and convective amplification alter levels and pitch observed on the ground. As the emissions propagate, they are influenced by spherical spreading, atmospheric absorption, and various ground effects.

In:

Lossless, narrowband source spectra

$L=L\left(f, \theta, M_{f}\right.$, altitude, throttle)

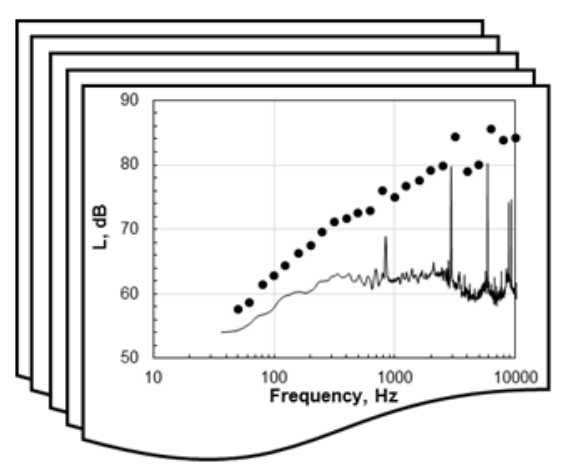

Installation effects:

- Source interactions

- Propulsion-airframe integration

Acoustic signature effects:

- Distance \& directivity vs. time impacts

- Airspeed, Doppler shift \& convective amplification

- Three engine throttle settings

Propagation effects:

- Spherical spreading

- Atmospheric absorption

- Ground reflections

- Lateral attenuation

- Pseudotones

Noise metric \& regulatory effects:

- Conversion to $1 / 3^{\text {rd }}$ octave band

- PNL noy-weighting

- PNLT tone correction penalties

- Event duration

- Three certification monitors
Out:

Certification EPNLs

- Lateral

- Flyover

- Approach

- Cumulative

Figure 2. System noise prediction schematically represented as an analytical modeling process.

If noise certification metrics for transport-category airplanes or jet airplanes are used as a measure of merit, additional complexities arise. Noise certification attempts to account for various psychoacoustic phenomena. Noise regulations are based upon the one-third octave band center frequency paradigm. Received noise levels are converted to perceived noise levels using noy-weighting factors. Particular emphasis is given to levels in the frequencies between $1000 \mathrm{~Hz}$ and $10,000 \mathrm{~Hz}$. An additional noise penalty is added if tones are present in the received spectra, with the highest penalties assigned to tones lying between $500 \mathrm{~Hz}$ and $5000 \mathrm{~Hz}$. 
During a noise certification test, spectral acoustic measurements are made as an airplane flies past three certification noise observation monitors on the ground (shown in Figure 3). Spectra are measured at half-second time intervals at each noise observation station. From these, tone-corrected perceived noise levels are computed. For transport-category large airplanes and for subsonic jetpowered airplanes of all sizes, the regulation metrics are cast in terms of the Effective Perceived Noise Level, or EPNL. The EPNL is an attempt to relate human annoyance to noise. It is a metric sensitive to frequency, tone content, and duration of a single airplane flyover event. EPNL is the metric regulated by ICAO and by the FAA for noise type certification. In noise certification parlance, the cumulative, or algebraic, sum of the three certification EPNLs is often used to capture all three measurements.

The community noise modeling process illustrated in

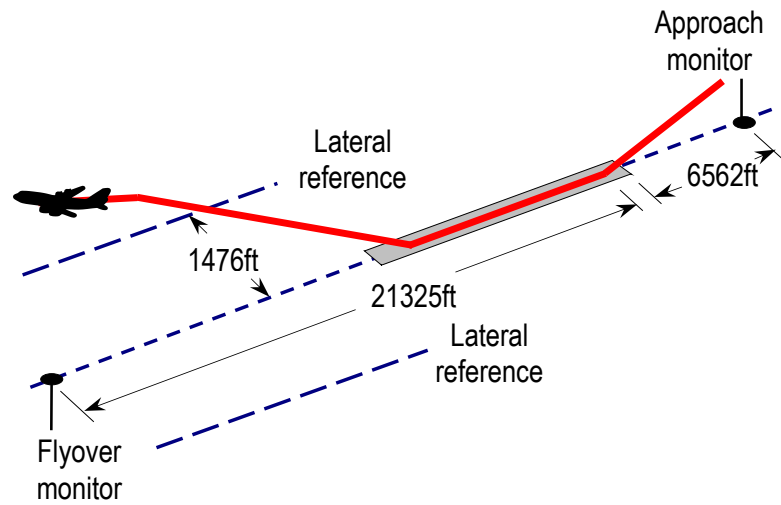

Figure 3. Noise certification monitor arrangement relative to takeoff and landing flight paths.

Figure 2 is an analytically tractable problem, solvable by physics-based system analysis tools. NASA's Aircraft Noise Prediction Program (ANOPP $[14,15])$ is an example of such a tool. In this study, ANOPP is given a noise source (suppressed by acoustic treatment), analytically flies it through the air, propagates it to an observer on the ground, and computes EPNLs.

\section{B. Candidate Suppression Models}

The suppression spectra are parameterized mathematically using a set of independent design variables. These "shape factor" variables are subject to manipulation by a multi-variable optimizer so that an objective function representing community noise is minimized. The shape factors are represented by a vector, $\mathbf{x}$, consisting of elements $x_{i}$. By this definition, a suppression spectrum can be represented by any number of simple mathematical expressions, as long as they faithfully characterize the shape of an actual suppression spectrum. A parameterized suppression spectrum is, in other words, a simplified metamodel or surrogate model of the actual suppression spectrum.

A simple example of a parameterized suppression spectrum might be given by a polynomial function of narrowband frequency. The narrowband linear bandwidth of frequency is arbitrary, as long as there is sufficient detail to describe the spectrum. Frequency could range from $45 \mathrm{~Hz}$ to $11,220 \mathrm{~Hz}$ : the boundaries of the one-third octave band defined by aviation regulations [16]. The coefficients of the polynomial would be the shape factor variables $x_{i}$, subject to optimization. At each pass of the optimizer, the calculated suppression would be subtracted from the known, untreated source, creating a suppressed source. Its levels would be converted to one-third octave band levels, analytically flown through the air, propagated to the ground, and an appropriate objective function would be computed.

But suppression levels need to be limited somehow, otherwise the "best" shape factors will be those that deliver ridiculously large and unrealistic levels of suppression. Unconstrained, an optimizer would drive overall suppression to very large values. One solution is to limit overall suppression by requiring a constant area to be enclosed by its spectrum. In other words, as shape factors $x_{i}$ are varied and optimized, the area bounded by the curve of the suppression spectrum over the frequency domain is required to remain constant. There is some physical basis to this, as liner wall impedance is finite. The tacit assumption is that the shape of the suppression spectrum is more important than its overall effectiveness, so that guidance can be given to liner designers.

Suppression can be limited by formulating the optimization problem with a constraint on enclosed suppression spectrum area. In that case, the burden of enforcing suppression limits is placed on the optimizer. Another approach is to design the suppression spectrum to always have a constant enclosed area. Statistical probability density functions can be used to characterize families of suppression curves, all having constant areas. Probability density functions inherently have constant enclosed areas, since the probability measure of an entire sample space is unity. Probability density functions naturally constrain the problem so that an optimizer can perform a simpler, unconstrained optimization. Probability density functions can be combined and modified to form quite complex shapes. This approach was emphasized in the previous paper [13].

Discretized suppression spectra are also possible. A suppression spectrum can be defined by discrete training points connected by spline functions. If their frequencies are defined, suppression levels become the $x_{i}$ to be varied by the optimizer. If many training points are used, shape control can be very precise, at the risk of burdening the optimizer with many optimization variables. Discretized suppression spectra were investigated in the previous study [13], but

4 of 13

American Institute of Aeronautics and Astronautics 
they performed poorly since most classical optimizers tend to perform best when there are as few design variables as possible. With the large number of training points required to define a suppression spectrum, the computational expense of evaluating finite difference gradients at every point was high. Many training points also were problematic for evolutionary and gradient-free search-strategy optimizers.

In this paper, however, discretized suppression spectra are used. This approach is enabled by analytic differentiation, and is discussed immediately below.

\section{Analytic Differentiation}

In the previous study [13], whenever a gradient-based optimizer was selected, local sensitivities were estimated using a finite difference formula for changes in the objective function with respect to perturbations of acoustic liner design variables. These design sensitivities (or sensitivity derivatives) were used to optimize the objective. This approach was used because finite difference sensitivities were easily and quickly implemented. But since many evaluations of the objective were necessary to compute all of the sensitivities, this approach proved to be computationally expensive when many optimization variables were considered. Lack of gradient precision due to numerical differentiation also contributed to wandering, poor convergence, and long optimization times. For these reasons, greatest success occurred when design variables were relatively few in number and when gradient-free optimizers were used. Even more success came when design variables were discretely enumerated and manipulated by an evolutionary optimizer.

The purpose of the present study is to apply analytic derivatives to a steepest-descent gradient optimization algorithm. Numerically precise sensitivities can be key to efficient convergence. In the previous study (and referring again to Figure 2), the objective evaluation model initially was viewed as a formidable "computational black box." However, the source code, internal variables and processes in ANOPP are known and well-documented. Further, the processes are explicit, deterministic, sequential, and without significant complicating loops or convergence iterations. Therefore, the noise propagation process (at least as modeled by ANOPP) is not a true black box. Excellent progress in penetrating this process has been made [17]. The noise propagation process can be reduced to a sequence of composite mathematical functions. Metrics and their derivatives are displayed in the tree diagram of Figure 4.

At the top of the Figure is the EPNL featured in the objective function and in noise regulations. It can be decomposed into the maximum tone-corrected perceived noise level (PNLT, given the notation $L_{T P N, \max }$ ) and the duration correction $D$ of the flyover event. In turn, PNLT is decomposed into the perceived noise level (PNL, given the notation $L_{P N}$ ) and the tone correction factor $C$. All of these noise metrics (formally defined by ICAO in [7]) are functions of proportional one-third octave band sound pressure levels (1/3OBSPL) received by a ground observer $L_{\text {rec }}(f)$. The received spectrum consists of 24 discrete sound pressure levels ranging from $50 \mathrm{~Hz}$ to $10 \mathrm{kHz}$. Further, a spectrum $L_{\text {rec }}(f)$ exists at each half-second interval as the airplane first approaches, and then recedes from, the noise measurement station. With some effort [17], the derivative of EPNL with respect to $L_{\text {rec }}(f)$ can be computed via analytic differentiation using the single- and multi-variable derivative chain rule. These derivatives have been incorporated into the next-generation ANOPP software, ANOPP2 [18], as callable application program interface routines.

With additional work, the received spectrum $L_{r e c}(f)$ at the noise measurement station can be followed up the propagation path to the airplane whence it was emitted. Correction terms for spherical spreading $\left(20 \log _{10} r / r_{r e f}\right)$, atmospheric absorption ( $r \alpha$, using the method required by ICAO, [19]), and ground reflections $\left(20 \log _{10} G\right.$, using the ANOPP method [20]) can be applied to the received spectrum to compute the emitted spectrum, $L_{e m}(f)$. The absorption $\alpha$ and ground reflection $G$ correction factors have a variety of dependencies, but for any given spectrum during the flyover event, the observer-airplane geometry and physical air and ground properties are known quantities. Thus, $\alpha$ and $G$ may be considered known, frequency-dependent parameters rather than true system variables. Results with respect to $L_{e m}(f)$ are as easily obtained as those with respect to $L_{\text {rec }}(f)$.

Further, the emitted one-third octave band spectra $L_{e m}(f)$ can be expressed as a function of emitted narrowband spectra $l_{e m}(f)$, which in turn are really the differences between hardwall $l_{e m, h w}(f)$ and suppression $l_{\text {supp }}(x, f)$ spectra. So in the end, though it might be tedious to derive and to program into software, the derivative of EPNL with respect to acoustic liner design variables $x_{i}$ may indeed be expressed analytically. But although tedious, the effort should pay off not only for a task such as this, but it should also enable better efficiency and accuracy in other optimization and design-of-experiment problems where certification noise is used in an objective function.

Reasonable simplifications can be made to this process. First, after examining all of the PNLTs of the flyover event, $L_{T P N, \max }$ could be singled out and minimized exclusively rather than the entire EPNL. The tacit assumption is that the design variables influence $L_{T P N, \max }$ by about as much as they influence $D$. This simplification is fair if the trajectory is fixed and if convective amplification, Doppler shift, and source directivity effects contribute equally to

5 of 13

American Institute of Aeronautics and Astronautics 
$D$ and to $L_{T P N, \max }$ as $x_{i}$ are varied. During the optimization process, all of the PNLTs of the flyover event could be examined occasionally to ensure the highest PNLT is always being optimized. This simplification relieves the optimizer from the burden of computing analytic derivatives for every spectrum on half-second intervals during the flyover event. Another simplification is to cast the design variables in terms of the one-third octave band spectra $L_{e m}(f)$ rather than narrowband spectra $l_{e m}(f)$. In this case, the optimizer is further relieved of considering the last derivative in the chain rule derivation, $\mathrm{d} L_{e m} / \mathrm{d} l_{e m}$. This simplification is fair if the source and suppression models can be accurately represented by coarser one-third octave band levels.

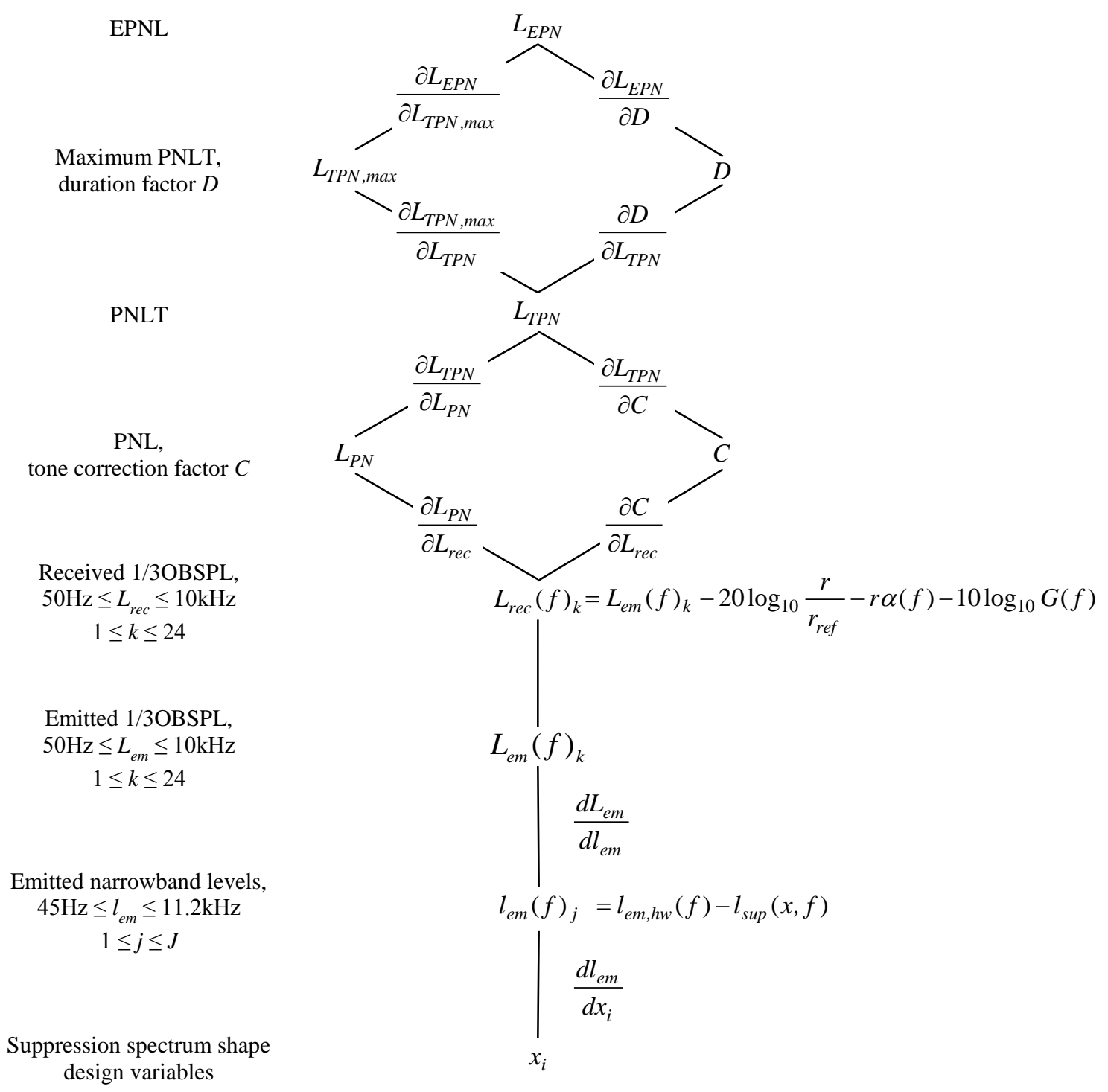

Figure 4. Noise metrics and sensitivities represented as a sequence of composite mathematical functions.

\section{Objective Function}

One possible community noise objective function is defined as

$$
O(\mathbf{x})=\sum_{i=1}^{I} w_{i} L_{E P N ~}(\mathbf{x})+R_{j} \max \left[0, \frac{A_{i n}(\mathbf{x})+A_{e x}(\mathbf{x})}{A_{i n, \max }+A_{e x, \max }}-1\right]^{2}
$$


$L_{E P N}$ is the notation for the EPNL certification noise metric. As written, $O(\mathbf{x})$ is a composite objective function consisting of the weighted sum of several EPNLs. Alternatively, the problem may be set up as a multiple-objective optimization in $L_{E P N}$, resulting in a Pareto-optimal solution (Indeed, a multi-objective optimization is used to estimate the benefit of variable-impedance liners, as discussed in Section III). In the special case where the weighting factors $w_{i}$ are unity and the number of observers $I=3, O(\mathbf{x})$ can represent the cumulative EPNL if the lateral, flyover, and approach certification monitors are properly located and modeled. Depending on designer's intent, $L_{E P N}$ may consist of suppressed sources only, or other unsuppressed noise sources on the airplane may also be added. When unsuppressed noise sources contribute to $L_{E P N}$, the relative contribution of the suppressed source is diminished, but it may more realistically portray the actual system noise.

The second term in Eq. (1) is an exterior additive dynamic penalty that penalizes infeasible solutions and drives the final result towards feasible space. $A_{i n}(\mathbf{x})$ and $A_{e x}(\mathbf{x})$ are the enclosed areas of the inlet and exit exhaust duct suppression spectra, respectively, which when added together may not exceed the sum of $A_{i n, \max }$ and $A_{e x, \max }$. In this context, the max operator results in the value of the constraint violation if it is positive, otherwise zero is returned. The penalty coefficient $R$ can be sensitive to and increase with $j$, the number of generations in an evolutionary optimization or with the number of solutions searched in a search-strategy optimization. Thus the severity of the penalty increases with the amount of the violation and with the number of successive iterations. When analytic differentiation is used to compute sensitivities, the gradient contributed by the penalty can be determined and added to the sensitivities computed by ANOPP2. In this study where discretized suppression spectra are used, enclosed area is calculated from the training points using the trapezoidal rule, and the derivative of $A$ with respect to $x$ is known. The steepest-descent optimizer always uses correct sensitivities, even when near the suppression limit.

\section{E. The OpenMDAO Model}

The OpenMDAO (version 1.71, 2016) frameworking software is used to model the process. OpenMDAO [21] is an open-source computing environment and frameworking tool developed by NASA for multidisciplinary systems analysis and optimization. OpenMDAO problems are coded in the Python scripting language. Components, groups and drivers are classes available in OpenMDAO to create objects. The objects are connected to form a sensible, multidisciplinary analysis of a problem. A collection of intrinsic filewrapping utilities are available for component classes to wrap external codes (in this case, ANOPP). The workflow of the noise model is shown in Figure 5.

The OpenMDAO model consists of two groups that may be executed independently. The objective evaluation group computes the value of the objective function, given hardwall source and suppression spectra. Inside this group, the hardwall source spectra to be suppressed is defined. The suppression spectra are determined from the independent shape variables, $x_{i}$. Using analytic derivatives, sensitivities with respect to the $x_{i}$ are computed by calling an application programming interface routine in ANOPP2 via Python. Finally, the objective evaluation component computes the suppressed source, applies flight effects, and, if necessary, converts narrowband to one-third octave band spectra. If EPNL is the objective function, an ANOPP input file is assembled from a template and run using filewrapping functions, EPNL is parsed from the ANOPP output, and returned to the group. The most computationally expensive part of the procedure is performed by ANOPP. The outer optimization group governs optimizer behavior, manipulation of the $x_{i}$, and calls the objective function evaluation. All of the component analyses are written in native Python code, except, of course, for the noise calculation performed externally by ANOPP.

OpenMDAO has drivers that support a variety of optimization methods. Included are classical methods such as gradient-based methods, one direct (search-strategy) method, and evolutionary and particle swarm methods. Selecting a successful optimizer is challenging for this kind of problem. In the original paper [13], greatest success occurred when design variables were relatively few in number and gradient-free optimizers were used. Gradient-based methods - with finite difference gradients - were less successful. It was hypothesized that the objective function was not continuous or beset by local optima, providing opportunities for gradient-based optimizers to become stuck. Also, if handicapped by an unhelpful starting point, suppression may attack a portion of the source spectrum far from its peak sound pressure level. For these reasons, it was not surprising that OpenMDAO's gradient-free Constrained Optimization by Linear Approximation (COBYLA) [22] driver performed well, at least when independent variables were allowed to be continuous-real. When discrete enumerated variables were chosen, the Pyevolve [23] evolutionary optimizer was successful (although it also performed well when variables were continuous-real).

In this study, optimization is performed by a simple multi-variable steepest-descent method. Suppression levels are set initially to zero. At every iteration, suppression levels at each discrete training point increase by amounts determined by gradients supplied via ANOPP2. Sensitivities are evaluated at every pass since analytic differentiation is computationally inexpensive. Indeed, this is recommended when tones are present, since sensitivities can vary dramatically at every pass and the frequency at which the tone correction penalty is evaluated often jumps from one

7 of 13

American Institute of Aeronautics and Astronautics 
bin to another. As the optimization progresses, the step size is reduced using a backtracking line search method. Optimization is complete when the suppression spectra converge on the suppression area constraint and when no further reductions in the objective value are returned.

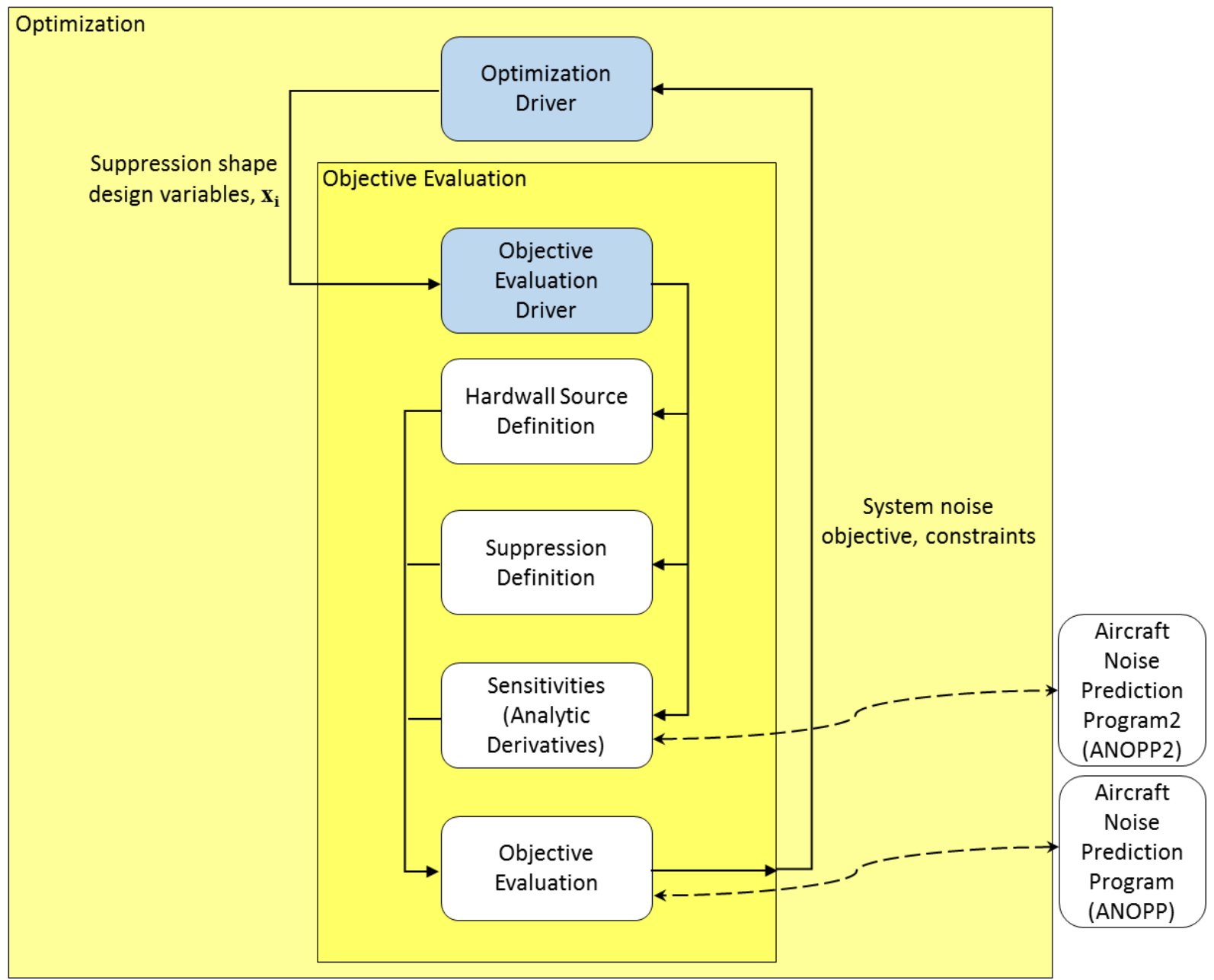

Figure 5. OpenMDAO model workflow.

\section{Results and Discussion}

The optimization method is applied first to a simple notional source flying past a single observer. The problem is deliberately simplified to verify and validate the process, and to determine the most successful optimization controls. It is also simple enough so that the optimum solution can be verified by inspection. Afterward, the method is used to estimate the benefit of variable-impedance liners using a more realistic hardwall fan source and a Pareto-optimal solution with three certification observers.

\section{A. Optimization of a Notional Source Spectrum}

An OpenMDAO model is developed to minimize the peak PNLT of a flyover event using a discrete suppression spectrum with 24 training points located on the one-third octave band center frequencies. The emitted, unsuppressed source spectrum at the beginning of the optimization is shown in Figure 6a. The unsuppressed emitted source (in red) consists of broadband content as well as a strong tone located at $1000 \mathrm{~Hz}$ and another tone at its harmonic, $2000 \mathrm{~Hz}$. 

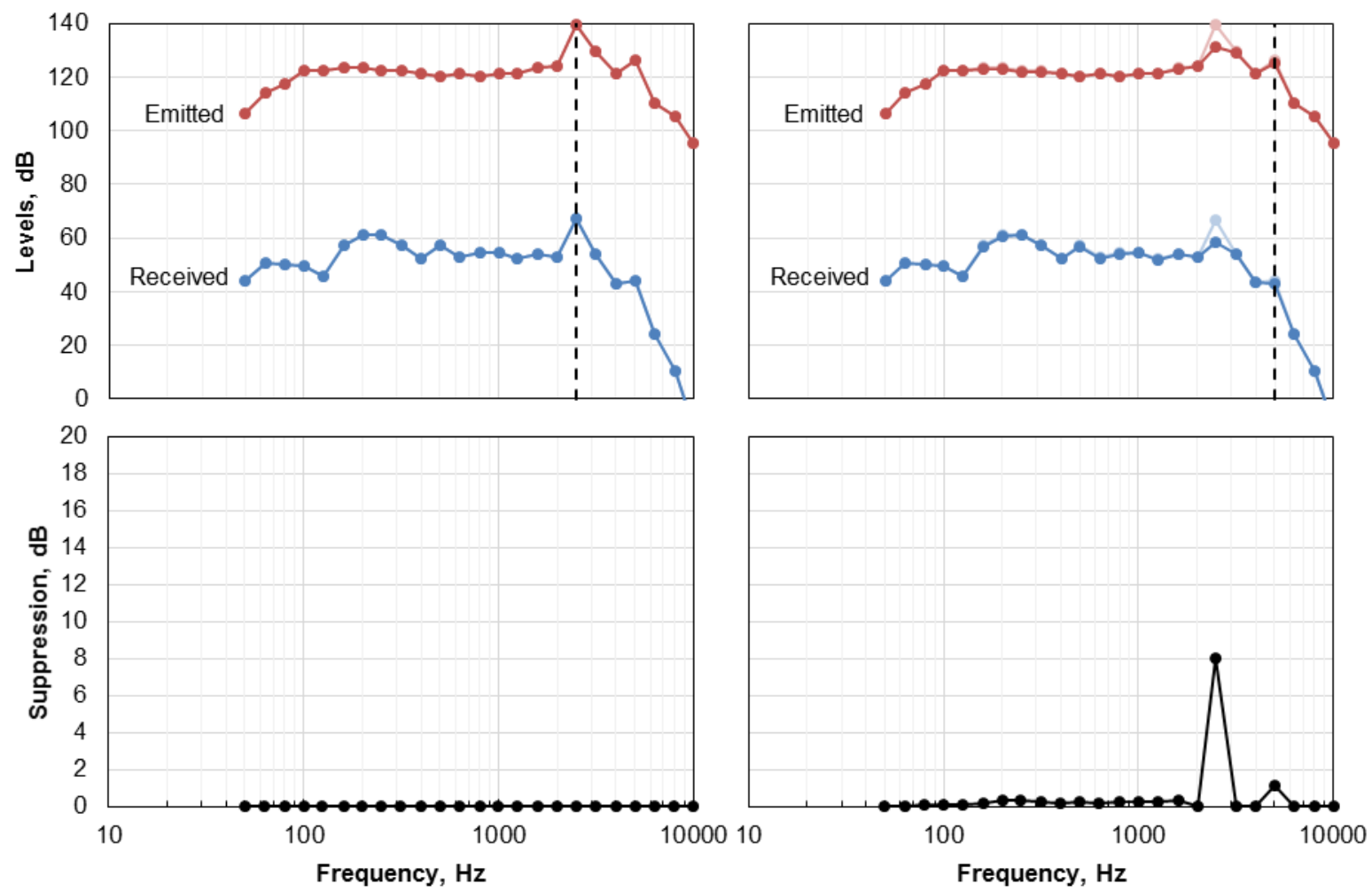

(a) Start, $L_{T P N}=88.2 \mathrm{~dB}, L_{P N}=83.7 \mathrm{~dB}$.

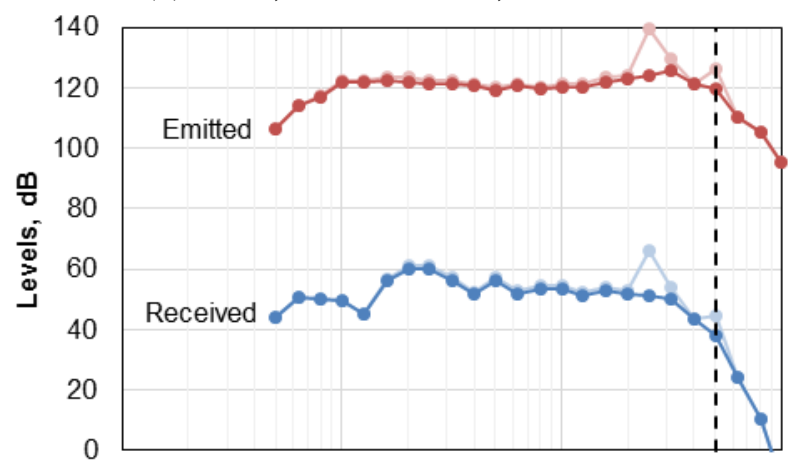

(b) Pass 8, $L_{T P N}=80.7 \mathrm{~dB}, L_{P N}=78.6 \mathrm{~dB}$.
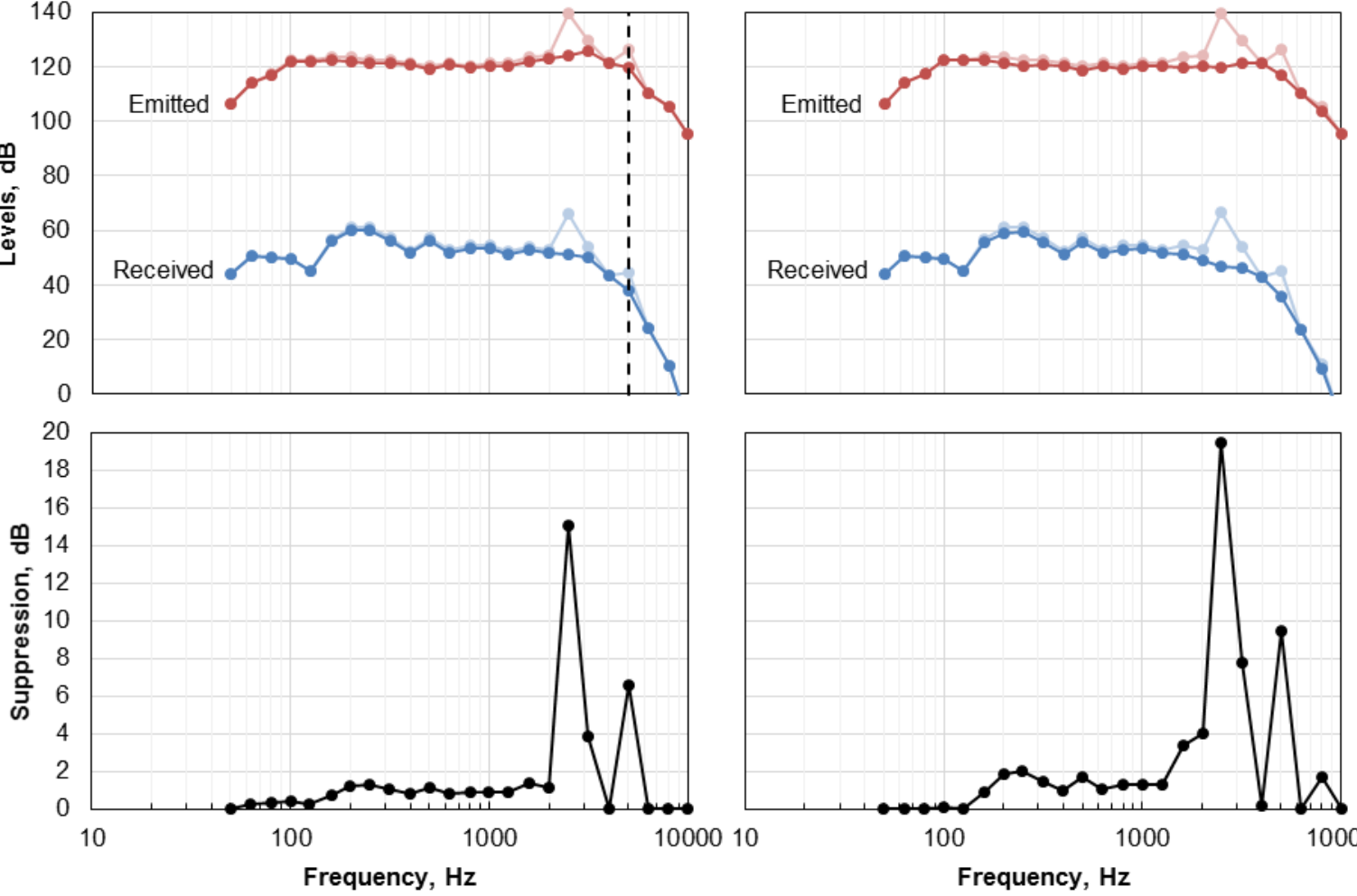

(c) Pass 30, $L_{T P N}=75.8 \mathrm{~dB}, L_{P N}=74.8 \mathrm{~dB}$.

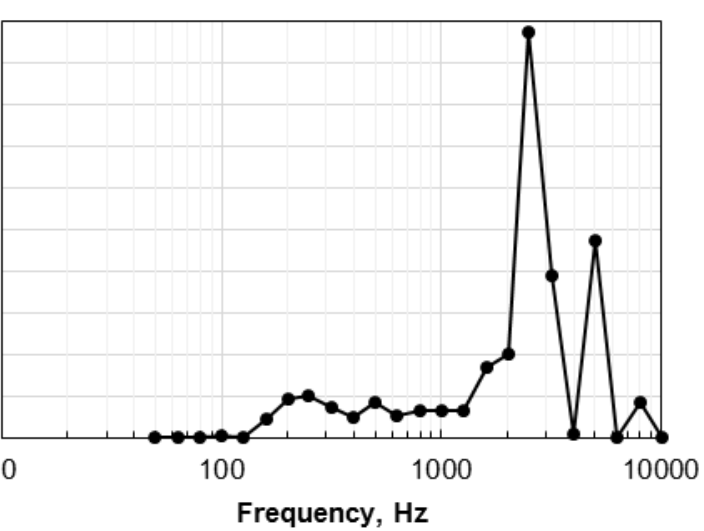

(d) Pass 111, $L_{T P N}=L_{P N}=72.5 \mathrm{~dB}$.

Figure 6. Optimization progress for notional spectrum test.

9 of 13

American Institute of Aeronautics and Astronautics 
The source is flown at an altitude of 1000 feet above ground elevation. A single lateral observer is located 1476 feet across from its flight path. The unsuppressed received spectrum is shown in blue in Figure 6a. Spherical spreading lowers the levels, as does atmospheric absorption, beginning mostly at frequencies above $1000 \mathrm{~Hz}$. Ground nulls and pseudotone irregularities produced by ground reflections are present (the receiver is located four feet above the ground), particularly at frequencies under about $800 \mathrm{~Hz}$ where ground effects are most efficient. Also shown in the upper chart is a vertical line indicating the frequency where the tone correction $C$ is evaluated. Regulations [24] permit tone correction penalties resulting from pseudotones to be ignored provided the tones can be shown to be artifacts of ground reflections unrelated to engine source noise. Thus in this problem, if a tone occurs at $800 \mathrm{~Hz}$ or less, $C$ is set simply to zero. The lower chart contains a plot of the suppression spectrum; initially set to zero. Unsuppressed, the received PNLT is 88.2TPNdB.

After eight iterations (Figure 6b), the optimizer has chosen to attack the fundamental tone by applying $8 \mathrm{~dB}$ of suppression at $1000 \mathrm{~Hz}$. Tone strength is low enough that $C$ is evaluated now at the harmonic. Very little emphasis has been given to broadband content suppression at this point. By 30 iterations (Figure 6c), some attention has been given to broadband suppression and the spectrum looks much like its final shape as it approaches its area equality constraint and the step size shrinks. By 111 iterations (Figure 6d), optimization is completed. Tones have been reduced such that there is no longer a tone correction penalty (i.e., $C$ is zero). The final PNLT is identical to the PNL at 72.5PNdB, with the notional liner providing more than $15 \mathrm{PNdB}$ of suppression.

\section{B. Estimated Benefit of Variable-Impedance Liners}

Analytic derivatives improve the efficiency and accuracy of gradient-based optimization methods and allow consideration of more design variables. These improvements to the method enable a practical investigation into the benefit of variable-impedance liners - a problem that previously was too timeconsuming to pursue before application of analytic derivatives.

The noise signature of a fan changes with engine power setting and flight condition, so hardwall noise is unique at each noise measurement station. Active and adaptive liners should be better equipped to tailor their impedance to a changing noise source than fixed-geometry liners. For fixed-geometry liners, an effective optimization strategy may be to minimize the cumulative EPNL. In that case, the objective function would be a composite function of the three certification EPNLs, as written in Eqn. 1 with the $w_{i}$ set to unity. But with variable liners, it would be fair to derive distinctly-different suppression spectra that minimize each of the three EPNLs individually. A comparison of these two optimal results - when tasked with suppressing a realistic hardwall fan noise source - would provide Table 1. Flight conditions.

\begin{tabular}{c|c|c|c}
\hline \hline Monitor & Fan speed, \% & Altitude, ft & Flight Mach \\
\hline \hline Lateral & 100 & 1000 & 0.27 \\
Flyover & 86 & 2400 & 0.28 \\
Approach & 62 & 394 & 0.21 \\
\hline \hline
\end{tabular}

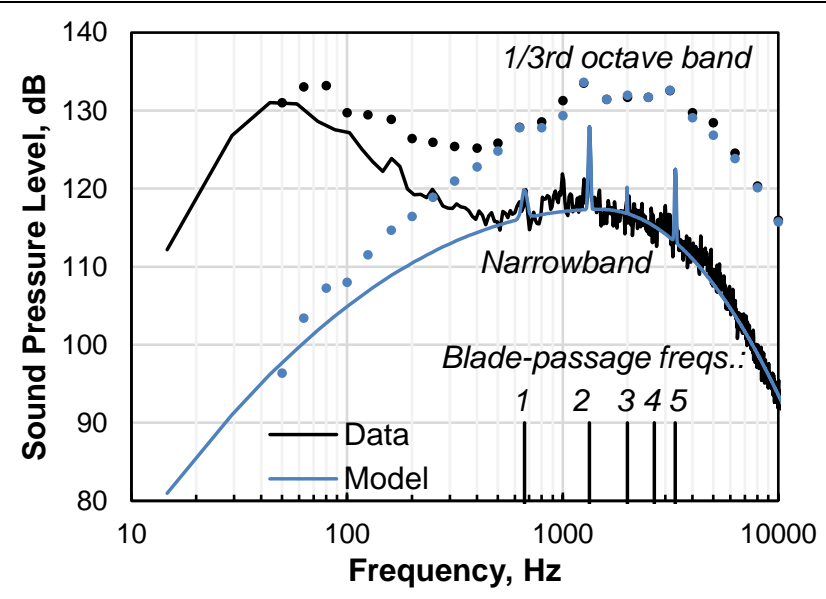

Figure 7. Measurements and surrogate model of scaled, static fan at $100 \%$ speed, polar angle 90deg.

an estimate of the benefit of variable liners (or perfectly-tunable variable liners, at any rate).

The hardwall noise source used is a 22-inch diameter model of Pratt \& Whitney's Advanced Ducted Propulsor [25]. With low tip speeds and a design pressure ratio of 1.29 , it is representative of modern, contoured, wide-chord fans used for high-bypass geared turbofans. The levels are adjusted from model-scale, 22-inch fan diameter to fullscale, 88-inch diameter by applying amplitude and frequency shifts using a linear scale factor of 4 . The data are further adjusted for convection from the wind tunnel Mach number of 0.10 to the flight conditions shown in Table 1. Data at three shaft speeds and flight conditions are considered, representing fan operation at the lateral, flyover and approach flight conditions used in certification. With these adjustments, the hardwall source noise is thought to be typical of a fan for an advanced narrow-body transport in the 737- or A320-class.

A mathematical model of static, full-scale, narrowband fan noise is developed to aid data manipulation. Using a noise surrogate model in place of measured spectra allows for removal of extraneous or spurious portions of the spectra that are not believed to be genuine fan noise, such as low-frequency airflow scrubbing and echoic facility noise

10 of 13

American Institute of Aeronautics and Astronautics 
sources. A full discussion of this process is described in the earlier report [13] and is not repeated here. An example of the surrogate modeling for one spectrum is shown in Figure 7.

Table 2. Optimized liner results, EPNdB.

\begin{tabular}{c|c|c|c}
\hline \hline Monitor & None & Fixed & Variable \\
\hline \hline Lateral & 87.8 & 81.8 & 78.2 \\
Flyover & 80.1 & 74.8 & 71.8 \\
Approach & 89.5 & 85.0 & 82.4 \\
Cumulative & 257.5 & 241.5 & 232.4 \\
\hline \hline
\end{tabular}

The model is executed with no suppression to determine the unsuppressed baseline. This result is shown in Table 2 and is identified as the nadir point in the multi-objective projection charts shown in Figure 8. Next, the model is coded to minimize the cumulative EPNL over all three certification observers. This is done by optimizing the shape of a single suppression spectrum. This solution represents the best performance a fixed-impedance liner can achieve. The result of this optimization is shown in Table 2 and is identified as the best fixed-impedance solution in Figure 8.

Last, distinctly-different suppression spectra are derived by minimizing the three certification EPNLs in multi-objective fashion. Variable constraints can be imposed on suppression levels to form Pareto frontiers of optimal results; a process mathematically equivalent to the epsilon-constraint method [26]. Projections of the frontiers are plotted in Figure 8. The best lateral, flyover and approach EPNLs are identified at frontier endpoints. The best fixed-impedance solution does indeed lie on the three-dimensional frontier, but it does not lie on the two-dimensional projections of the frontier shown in the Figure. Several gaps are present in the frontiers. These are due to the discontinuous behavior of $\partial C / \partial L_{\text {rec }}$ on rare occasions.

Presenting Pareto-optimal frontiers for this problem, however, is a bit disingenuous. Flying past the three certification monitors are separate events. A true adaptive liner would have time to adjust its impedance to something appropriate for the condition (indeed, this is entirely the point). Therefore, the correct ideal performance is not a frontier at all, but rather the utopian points identified in the Figure.

In any case, with these assumptions, the benefit of a variable-impedance liner relative to a fixed-geometry liner can be estimated to be approximately $3 \mathrm{EPNdB}$ at each observation monitor, or approximately $9 \mathrm{EPNdB}$ on a cumulative basis. The benefit, of course, would be less if other unsuppressed noise sources are included.
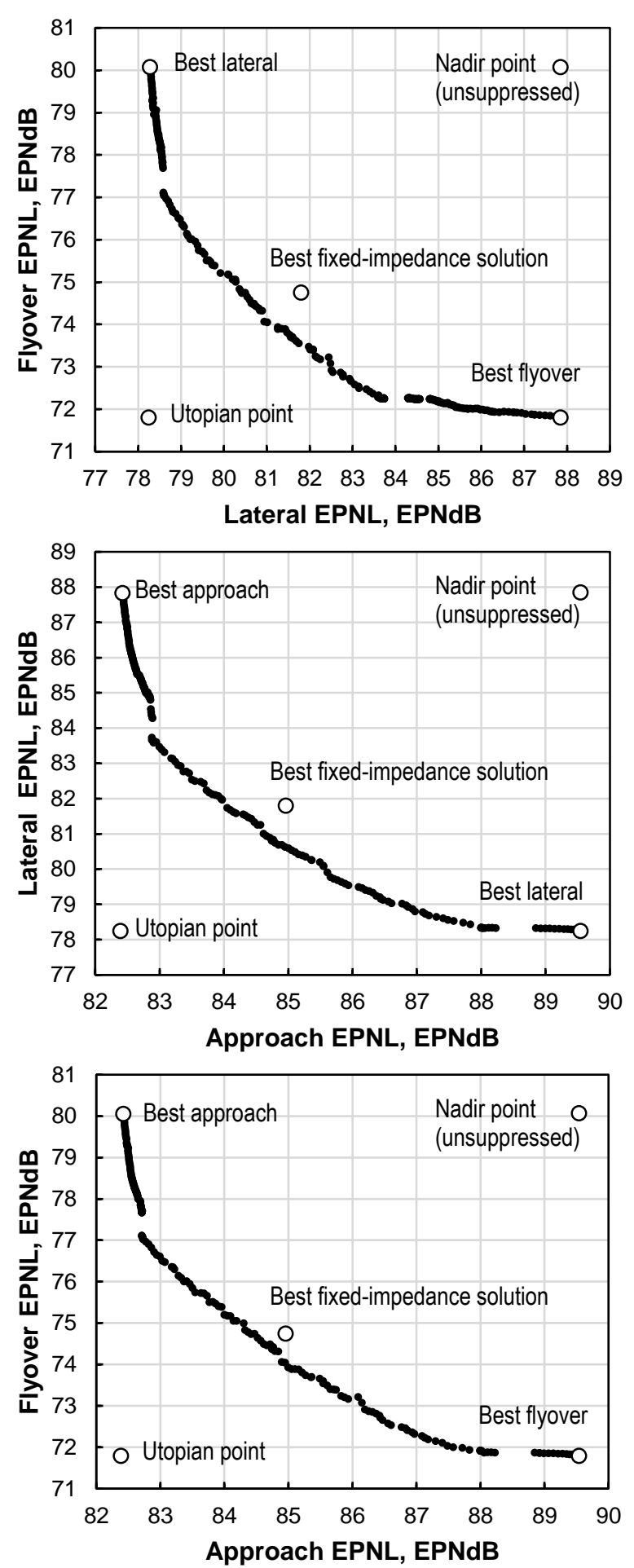

Figure 8. Optimal variable-impedance solutions compared to the fixed-impedance optimum.

11 of 13

American Institute of Aeronautics and Astronautics 


\section{Conclusions}

Given a hardwall noise source to suppress with acoustic treatment, there exists an ideal suppression spectrum shape that minimizes noise perceived by observers on the ground. But characteristics of that spectrum can be difficult to know. An analytical method is developed that derives the shape characteristics of the ideal target attenuation spectrum. The method requires mathematically parameterizing the suppression spectrum such that it is represented by a set of independent "shape factors," which are design variables manipulated by an optimizer. Once the ideal shape is known, the geometric design and the impedance characteristics of a real acoustic liner can be derived to match it. The method is written using the OpenMDAO frameworking software developed by NASA for multidisciplinary systems analysis and optimization. Analytic derivatives provide precise gradients and replace finite difference gradients used in a previous paper. In addition, the benefit of variable impedance liners is explored using a multi-objective optimization. Relative to a fixed-geometry liner, the cumulative benefit of a notional variable-impedance liner is estimated to be 9EPNdB.

\section{Acknowledgments}

Thanks to NASA's Advanced Air Transport Technology Project and Transformational Tools and Technologies Project for supporting this study.

\section{References}

[1] Parrott, T.; and Jones, M.: “Assessment of NASA's Aircraft Noise Prediction Capability, Chapter 6: Uncertainty in Acoustic Liner Impedance Measurement and Prediction,” NASA/TP-2012-215653.

[2] Rice, E. J., "Attenuation of Sound in Soft-Walled Circular Ducts," NASA TMX-52442, Jan. 1968.

[3] Lester, H. C., and Posey, J. W., "Duct liner optimization for turbomachinery noise sources," NASA TMX-72789, Nov. 1975.

[4] Rice, E. J., "Optimum Wall Impedance for Spinning Modes - A Correlation with Mode Cutoff Ratio," Journal of Aircraft, Vol. 16, No. 5, 1979, pp. 336-343; also AIAA Paper 78-193, Jan. 1978. doi: $10.2514 / 3.58528$

[5] Lester, H. C., Preisser, J. S., and Parrott, T. L., "Design and Flight Test of a Kevlar Acoustic Liner," Journal of Aircraft, Vol. 21, No. 7, 1984, pp. 491-497; also AIAA Paper 83-0781, Apr. 1983. doi: $10.2514 / 3.44998$.

[6] Gaeta, R. J., Jr., and Ahuja, K. K., “A Tunable Acoustic Liner,” AIAA Paper 1998-2298, June 1998. doi: 10.2514/6.1998-2298.

[7] “Annex 16 to the Convention on International Civil Aviation, Vol. I: Aircraft Noise," International Standards and Recommended Practices - Environmental Protection, 7th ed., International Civil Aviation Organization, Montreal, July 2014.

[8] "Noise Standards: Aircraft Type and Airworthiness Certification," U.S. Code of Federal Regulations, Federal Aviation Advisory Circular 36-4C, 2003, Title 14, Chap. 1, Part 36.

[9] Zlavoq, G. and Eversman, W., "A Statistical Approach to Optimum Design of Inlet Acoustic Treatment,” AIAA Paper 20062523, May 2006. doi: $10.2514 / 6.2006-2523$.

[10] Lafronza, L., McAlpine, A., Keanne, A. J., and Ashley, R. J., "Response Surface Method Optimization of Uniform and Axially Segmented Duct Acoustics Liners," Journal of Aircraft, Vol. 43, No. 4, 2006, pp. 1089-1102. doi: 10.2514/1.17727.

[11] Copiello, D., and Ferrante, P., "Multi-Objective Optimization of 'True' Zero-Splice Liners for Aero-Engine Intakes,” AIAA Paper 2009-3107, May 2009. doi: 10.2514/6.2009-3107.

[12] Copiello, D., Ferrante, P., Iemma, U., and Maci, A., "Preliminary Design of Aero-Engine Intake Acoustic Liners by Means of the Multi-Objective Approach," AIAA Paper 2010-3828, June 2010. doi: $10.2514 / 6.2010-3828$.

[13] Berton, J. J. : "Requirements-Driven Optimization Method for Acoustic Treatment Design," manuscript accepted for publication, J. Aircraft, forthcoming; also AIAA Paper 2016-2789, June 2016.

[14] Gillian, R. E.: “Aircraft Noise Prediction Program User's Manual,” NASA TM-84486, 1983.

[15] Zorumski, W. E.: “Aircraft Noise Prediction Program Theoretical Manual, Parts 1 and 2,”NASA TM-83199, 1982; Currently maintained at NASA Langley by the ANOPP team in electronic format and provided upon request; Latest revision: Level 31.

[16] International Electrotechnical Commission, "Octave, Half-Octave, and Third Octave Band Filters Intended for the Analysis of Sounds and Vibrations," IEC Publication No. 225-1966, 1966.

[17] Lopes, L. V., "Robust Acoustic Objective Functions and Sensitivities in Adjoint-Based Design Optimizations and the Propagation of Uncertainty in Noise Computations," AIAA Paper 2017-1673, Jan. 2017.

[18] Lopes, L. V. and Burley, C. L., "Design of the Next Generation Aircraft NOise Prediction Program: ANOPP2," AIAA Paper 2011-2854, June 2011.

12 of 13

American Institute of Aeronautics and Astronautics 
[19] Society of Automotive Engineers: "Standard Values of Atmospheric Absorption as a Function of Temperature and Humidity," Aerospace Recommended Practice 866A, 1975.

[20] Chien, C. F., and Soroka, W. W.: "Sound Propagation Along an Impedance Plane," Journal of Sound and Vibration, Vol. 43, No. 1, Nov. 8, 1975, pp. 9-20.

[21] Gray, J., Moore, K. T., Hearn, T. A., and Naylor, B. A., "Standard Platform for Benchmarking Multidisciplinary Design Analysis and Optimization Architectures," AIAA Journal, Vol. 51, No. 10, Oct 2013, pp. 2380-2394.

[22] Powell, M. J. D.: “A Direct Search Optimization Method that Models the Objective and Constraint Functions by Linear Interpolation", Advances in Optimization and Numerical Analysis, eds. S. Gomez and J-P. Hennart, Kluwer Academic (Dordrecht), 1994, pp. 51-67.

[23] Perone, C.S.: "Pyevolve: A Python Open-Source Framework for Genetic Algorithms," SIGEVOlution, vol. 4, no. 1, pp. 1220, 2009.

[24] "Environmental Technical Manual, Vol. I, Procedures for the Noise Certification of Aircraft," International Civil Aviation Organization (ICAO), Committee on Aviation Environmental Protection, 2nd ed., Document 9501, 2015.

[25] Dittmar, J. H., Elliott, D. M., and Bock, L. A., "Some Acoustic Results from the Pratt and Whitney Advanced Ducted Propulsor - Fan 1,” NASA TM-1999-209049, Mar. 1999.

[26] Haimes, Y. Y., Lasdon, L. S., and Wismer, D. A., "On a Bicriterion Formulation of the Problems of Integrated System Identification and System Optimization," IEEE Transactions on Systems, Man and Cybernetics, Vol. 1, No. 3, 1971, pp. 296297.

13 of 13

American Institute of Aeronautics and Astronautics 